\title{
Ballistic Focusing of Polyenergetic Protons Driven by Petawatt Laser Pulses
}

\author{
S. Kar, ${ }^{1}$ K. Markey, ${ }^{1,2}$ M. Borghesi, ${ }^{1}$ D. C Carroll, ${ }^{3}$ P. McKenna,${ }^{3}$ D. Neely, ${ }^{2}$ M. N. Quinn, ${ }^{3}$ and M. Zepf ${ }^{1, *}$ \\ ${ }^{1}$ Centre for Plasma Physics, School of Mathematics and Physics, Queen's University Belfast, BT7 1NN, United Kingdom \\ ${ }^{2}$ Central Laser Facility, Rutherford Appleton Laboratory, OX11 OQX, United Kingdom \\ ${ }^{3}$ SUPA, Department of Physics, University of Strathclyde, Glasgow, G4 ONG, United Kingdom
}

(Received 19 September 2010; published 2 June 2011)

\begin{abstract}
By using a thick $(250 \mu \mathrm{m})$ target with $350 \mu \mathrm{m}$ radius of curvature, the intense proton beam driven by a petawatt laser is focused at a distance of $\sim 1 \mathrm{~mm}$ from the target for all detectable energies up to $\sim 25 \mathrm{MeV}$. The thickness of the foil facilitates beam focusing as it suppresses the dynamic evolution of the beam divergence caused by peaked electron flux distribution at the target rear side. In addition, reduction in inherent beam divergence due to the target thickness relaxes the curvature requirement for short-range focusing. Energy resolved mapping of the proton beam trajectories from mesh radiographs infers the focusing and the data agree with a simple geometrical modeling based on ballistic beam propagation.
\end{abstract}

The observation of an energetic proton beam (of several tens of $\mathrm{MeV}$ ) from the interaction of intense laser pulses with solid targets has opened up new avenues of research for the pursuit of widespread applications in health care, industry, and scientific research [1]. One of the key issues with the energetic particle beam is its inherent large divergence which poses significant design constraints on many of its potential applications, for instance, the envisioned proton driven fast ignition (FI) scheme [2]. In this scheme the optimum stand-off distance, which is about a few $\mathrm{mm}$, of the proton source from the hot spot is a trade-off between the FI target design and temporal dispersion of the polyenergetic ion pulse. Because of typical efficiency less than $10 \%[3,4]$ of the ion acceleration mechanism, it is therefore crucial to maintain the ion flux over mm scale in order to fulfill the driver energy requirement for ignition. Several methods have been demonstrated so far in order to reduce the beam divergence of a selected narrow part of the proton spectrum. They mainly employ permanent magnet quadrupoles [5-7] or a compact ion lens driven by a secondary laser beam [8], and the shaped target scheme exploiting the target self-charging during interaction [9]. However, it is not clear yet how these schemes could be extrapolated for a desirable performance in a FI scenario, where one would require the full flux of the beam to be concentrated at a small (order of $\mathrm{mm}$ ) distance from the source.

The ballistic focusing scheme using curved targets (first proposed by Wilks et al. [10]) allows, in principle, the full beam (achromatic) focusing down to a small spot size, thanks to the extremely low beam transverse emittance [11]. The potential of the scheme has been demonstrated in experiments $[12,13]$, where the focused proton beam produced at moderate $\left(10^{18-19} \mathrm{~W} \mathrm{~cm}^{-2} \mu \mathrm{m}^{2}\right)$ laser irradiance was capable of heating metal foils isochorically to several tens of $\mathrm{eV}$, mainly by a narrow band of energies around a few $\mathrm{MeV}$. The experimental work presented here investigates the focusing effect of foil curvature on proton beams at a considerably higher laser intensity or proton energy regime, relevant to proton fast ignition and postacceleration schemes. One-dimensional focusing of protons at energies above $10 \mathrm{MeV}$ (up to $\sim 25 \mathrm{MeV}$ ) driven by a petawatt laser has been demonstrated for the first time. We report substantial progress here in terms of demonstrating clear "ballistic" and achromatic focusing of high energy protons while preserving the laminarity of the beam - which allows highly focused beams to be obtained from an optimized target geometry. The quality of the achieved beam shaping is inferred by energy resolved mapping of differential beam characteristics from a onedimensionally curved target and is reproduced by geometrical modeling. The observed achromatic focusing of $10-25 \mathrm{MeV}$ protons to radii as small as $25 \mu \mathrm{m}$ at a distance of $\sim 1 \mathrm{~mm}$ from the target $(250 \mu \mathrm{m}$ thickness and $350 \mu \mathrm{m}$ radius of curvature) provides the level of target design flexibility that is important to the success of the proton driven fast ignitor.

The data were collected in an experiment conducted at the Rutherford Appleton Laboratory, employing the

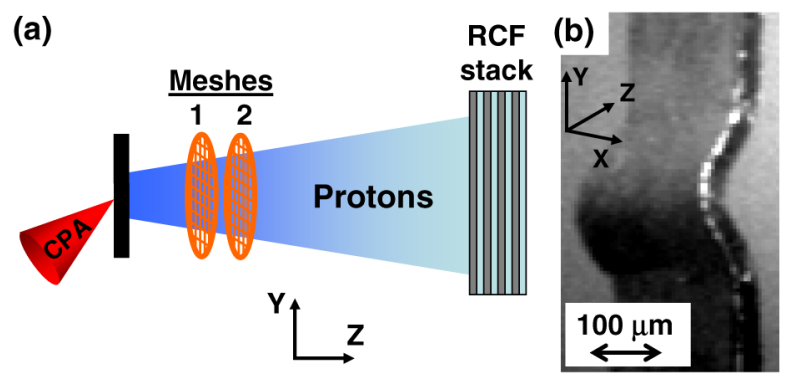

FIG. 1 (color online). (a) Side view of the experimental setup drawn schematically. (b) A photograph of the hemicylindrical target, of $350 \mu \mathrm{m}$ radius of curvature, fielded in the experiment. 


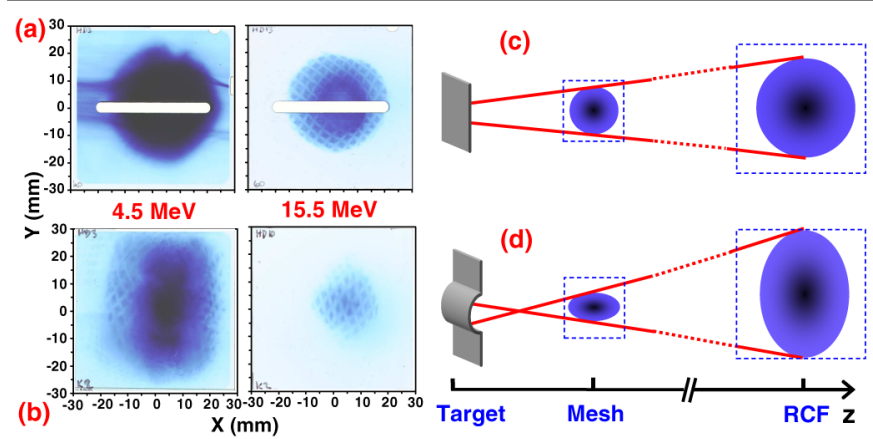

FIG. 2 (color online). Proton beam profiles obtained experimentally for (a) reference flat foil and (b) hemicylindrical targets for two different energies. The blank areas in the dose distribution in Fig. 2(a) correspond to horizontal slots cut in the RCFs in order to allow the proton beam to be diagnosed by complementary Thompson spectrometers. (c) and (d) are the schematics showing the proton beam longitudinal envelope and observed beam transverse profiles at different planes (viz., at meshes and the RCF) for flat foil and hemicylindrical targets, respectively.

Vulcan petawatt laser delivering $350 \mathrm{~J}$ energy in pulses of $\sim 750 \mathrm{fs}$ FWHM duration. The peak intensity on the target reached $\sim 4 \times 10^{20} \mathrm{~W} \mathrm{~cm}^{-2}$ by $f / 3$ focusing of the laser. A schematic of the experimental setup is shown in Fig. 1(a). Two types of targets were fielded, viz., reference flat foil targets and hemicylindrical targets [Fig. 1(b)]. The targets were made of $250 \mu \mathrm{m}$ thick Sn foil. The radius of curvature of the hemicylindrical target was $350 \mu \mathrm{m}$ over the $Y Z$ plane (henceforth referred to as the focusing plane) and infinite (i.e., a flat surface) in the orthogonal nonfocusing $(X Z)$ plane. Two periodic meshes of 400 (mesh-1) and 200 (mesh-2) lines-per-inch were placed in the proton beam at a distance of 1.25 and $2.25 \mathrm{~mm}$, respectively, from the target rear surface. The mesh radiographs, produced via multiple small angle scattering of protons in the mesh grids [14], recorded by stacks of radiochromic films
(RCFs) enabled energy resolved mapping of beam trajectories.

As shown in Fig. 2(a), the proton beam from the reference flat foil target showed a uniform and circular spatial profile for all proton energies (up to $33 \mathrm{MeV}$ ) detected by the stacks of RCFs. The shape and size of the mesh structure also remained fairly uniform across the whole beam profile. In contrast, a significant difference in the divergence of the beam and magnification of the mesh in the RCF is observed between the focusing and nonfocusing planes for the case of hemicylindrical targets [see Fig. 2(b)]. While the proton beam divergence over the nonfocusing plane is similar to that of the reference flat foil case, over the focusing plane significantly larger divergence is measured with higher mesh magnification. Looking at the beam cross section over the mesh-2 plane [see Fig. 3(a)], we found that the size of the proton beam (measured by counting the number of mesh periods in the beam over the $\mathrm{RCF}$ ) in the focusing plane is significantly smaller than in the nonfocusing plane [see the schematics shown in Figs. 2(c) and 2(d)].

In order to substantiate the observed behavior of the beam, the location of the virtual source (VS) [11] for different proton energies was estimated by extrapolating the final beam envelope, constructed by joining beam sizes over the RCF and mesh-2 planes, along the beam propagation axis towards the target. As expected over the nonfocusing plane of targets with one-dimensional curvature, the trend of VS positions with increasing proton energy remains in line with the characteristics of the planar reference foil case [see Fig. 3(b)]. On the other hand, a clear deviation in the VS characteristics is observed over the focusing plane of the hemicylindrical target. As shown in Fig. 3(b), the VS of the proton beam in the focusing plane is located at an unusually large distance of $1 \mathrm{~mm}$ from the target rear surface, which explains the differential
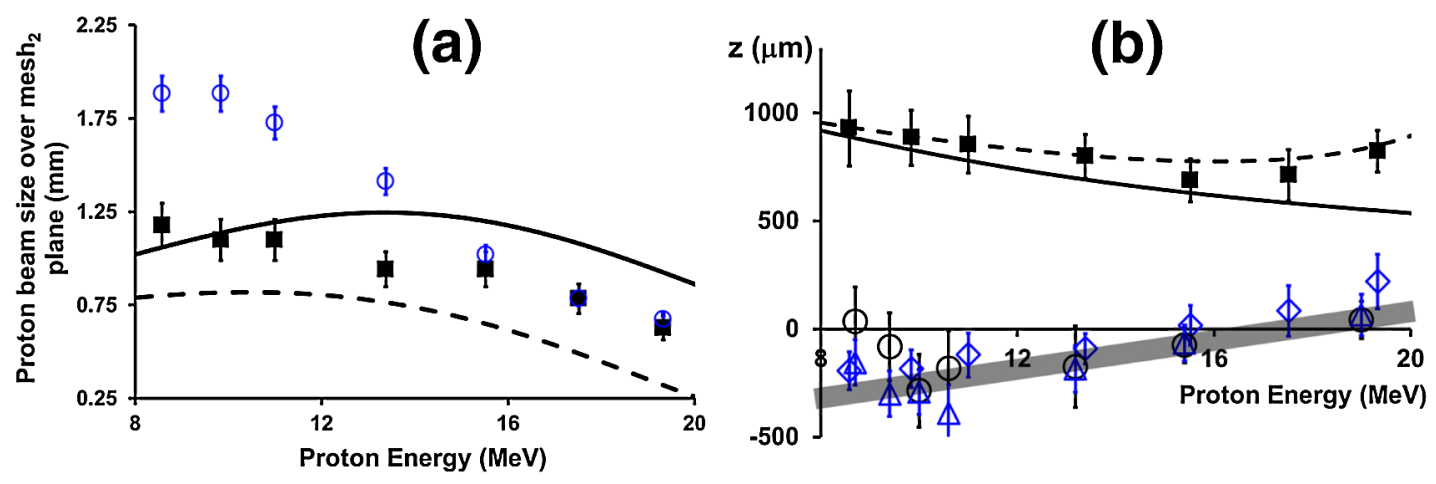

FIG. 3 (color online). (a) Data points showing beam transverse size at the plane of mesh-2 over focusing (black squares) and nonfocusing (blue circles) planes of the hemicylindrical target. (b) Graph showing beam VS positions for different energies from the hemicylindrical target for focusing (black squares) and nonfocusing (black circles) planes; reference flat foil target across two orthogonal planes (blue diamonds and triangles). The thick gray line illustrates that the positions of the VS for the nonfocusing plane of the hemicylindrical target remain in line with the characteristics of the planar reference foil case. Black dashed and solid curves in both (a) and (b) represent the respective results obtained from the simple geometrical curvature modeling for the hemicylindrical target by considering lower and upper limit profiles, respectively, of proton source sizes shown in Fig. 4(c). 
magnification of the mesh bars (higher magnification over the focusing plane) observed in the RCF. The beam divergence measured from this VS position is noticeably larger than the beam divergence from the planar reference foil. Thus the observed beam envelope can only be explained as a result of ballistic focusing of protons at the measured VS position.

The characteristics, such as divergence and transverse evolution, of the proton beam emergence from target rear surface is central to achieve focusing by a small radius of curvature (of a few hundreds of microns as required for a beam of $\sim 50^{\circ}$ divergence). Even after the acceleration, the beam divergence is strongly affected by plasma effects close to the target (a few hundreds of microns depending on laser irradiation, as diagnosed by the associated electric field at the plasma front in Ref. [15]). Thus the dynamics of the beam divergence depends on the shape of the plasma front at the beginning of free propagation, which in turn depends upon the hot electron distribution across the target rear surface producing the accelerating sheath [16]. Since the emergence of a proton beamlet is defined by the local normal to the sheath, an expanding plasma front (isodensity contour at a given time) of Gaussian shape [15-17] will give a curved trajectory to the beam emerging from the wings (the area beyond the inflection point) of the sheath, as shown schematically in the Fig. 4(a). By suppressing the hot electron refluxing the wings of the Gaussian ion front can be suppressed (as demonstrated in the Ref. [17]), and will allow the whole beam to emerge ballistically. Refluxing can be significantly constrained by increasing target thickness. For example, in the case of the thick $(250 \mu \mathrm{m})$ target used in our experiment, the transit time of hot electrons (moving with a speed close to that of light) for a single pass is similar to the incident laser pulse duration. Moreover, the transverse spreading of the hot electrons while refluxing about the thick target will heavily suppress the wings (or even truncate them due to the ionization threshold of the contaminants), eventually forming a flatter flux distribution at the target rear than produced in a thin target case. Indeed, as can be seen by comparing the divergence characteristics for two different ion front shapes shown in the Ref. [17] with the divergence characteristic shown in Fig. 4(b), the $250 \mu \mathrm{m}$ thick target resembles closely the case of an inverse parabolic or truncated Gaussian plasma front.

The focusing scheme using thick targets results in an additional advantage - the smaller cone angle of the beam produced from a thick target [for example, there is a reduction of more than $20^{\circ}$ for the $250 \mu \mathrm{m}$ thick target in comparison to a $25 \mu \mathrm{m}$ thick target, as shown in Fig. 4(b)] relaxes the curvature requirement for a given focal length.

Considering the ballistic emergence of proton beamlets from the thick target, the experimental results are reproduced by a simple geometrical model. In this model the
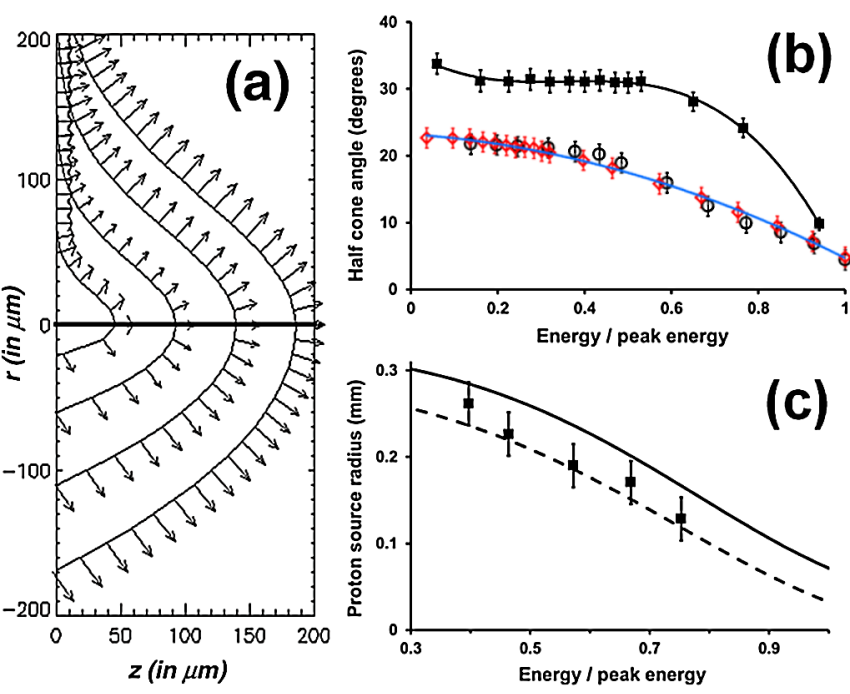

FIG. 4 (color online). (a) Schematic (not to scale) showing nonballistic (upper half) and ballistic (lower half) emergence of proton beamlets from an expanding ion front of Gaussian and parabolic shapes, respectively. The arrows show the direction of local normal to the ion fronts. (b) Half cone divergence angle of the proton beam from $25 \mu \mathrm{m}$ thick Al target (black squares), from $250 \mu \mathrm{m}$ thick (red diamonds) plain foil, and across the nonfocusing plane of the hemicylindrical target (black circles). (c) Proton source sizes $r(E)$ for different energies obtained from the reference shot. It is estimated by extrapolating a linear beam envelope, defined by the beam size at the mesh planes, back to the target rear surface. Black dashed and solid curves represent the profiles used in the modeling attributing to lower $\left[r(E)_{\min }\right]$ and upper $\left[r(E)_{\max }\right]$ limits, respectively. Since the peak proton energies of the hemicylindrical and planar shots were different (23 MeV and $33 \mathrm{MeV}$, respectively) due to shot to shot variations, $\theta_{i}(E)$ and $r(E)$ are plotted against energy normalized to the peak proton energy.

change in beam direction at any point over the curved rear surface of the target is formulated as the rotation of the local target normal due to the curvature-i.e., the change in angular divergence, $\triangle \theta(E)=\left|\frac{\pi}{2}-\tan ^{-1}\left(\left.\frac{d f}{d y}\right|_{r(E)}\right)\right|$, where $f(y)$ is the target rear surface curvature and $r(E)$ is the source radius of the protons of energy $E . f(y)$ was obtained by using a white light interferometer in order to map the curvature accurately. Across the focusing plane of the target, $f(y)$ is approximately circular (of radius of curvature of $0.35 \mathrm{~mm}$ ) in the central region and Gaussian in the periphery. The inherent divergence of the proton beam $\left[\theta_{i}(E)\right]$ and $r(E)$ is obtained from the reference flat foil target, which is shown in Figs. 4(b) and 4(c). As shown in Figs. 3(a) and 3(b), the experimental data points are in a good agreement with the geometrical modeling and fall within the simulated profiles corresponding to reasonable upper and lower limits of the estimated $r(E)$ [see $r_{\text {min }}(E)$ and $r_{\max }(E)$ in Fig. 4(c)].

An upper limit for the beam size at the focal plane is estimated by ray tracing of mesh imprints-the waist radius ranges from $\sim 90$ to $\sim 25 \mu \mathrm{m}$ over the proton energy 
spanning from 10 to $20 \mathrm{MeV}$, respectively. It is to be noted that an ideal point focus for the beam due to ballistic focusing was not expected in our case due to the noncircular curvature of the target and finite intrinsic emittance of the beam. The mesh radiographs also reveal no significant change in local emittance (for $18 \mathrm{MeV}$ protons it is estimated as $0.12 \pm 0.07 \pi \mathrm{mm}$ mrad) across the proton beam when comparing the flat foil and hemicylindrical target cases. This is expected in our case as the comoving electrons keep traveling with the protons during the divergence manipulation by the target curvature, unlike other focusing schemes $[5,8,9]$ where electric or magnetic fields are used to bend the proton trajectories. Therefore, with optimized target parameters the curvature scheme will allow the quasilaminar beam to focus down to the smallest spot possible without any degradation due to Coulomb explosion.

The conversion efficiency from laser to protons above $4 \mathrm{MeV}$ for the case of $250 \mu \mathrm{m} \mathrm{Sn}$ flat foil is found to be $1.5 \% \pm 0.5 \%$. This is still substantial but somewhat less than the typical values of around 5\% reported for targets of tens of microns thickness [3,4]. This reduction is expected due to electron transport losses and the suppression of electron refluxing. It is worth noting that no particular effort was made to optimize the proton flux, e.g., by finding the optimum trade-off between thickness and focusing or by varying the target $Z$. In principle, the conversion efficiency from thick targets can also be improved by increasing laser-to-electron conversion [18-21]. For most applications (such as isochoric heating) the small reduction in laser-to-proton conversion efficiency is more than compensated by the improved quality and achromaticity of the focus. In terms of maximum proton energy that can be achieved, the increased thickness of the target does not result in a significant reduction of the maximum energy, in agreement with previous work by Yuan et al. [22]. For example, $10-30 \mathrm{MeV}$ is an optimal range for the proton fast ignition scheme [2] and is produced in our case.

In conclusion, employing a $250 \mu \mathrm{m}$ thick foil with $350 \mu \mathrm{m}$ radius of curvature polyenergetic protons was seen to be focused ballistically at a distance of $\sim 1 \mathrm{~mm}$ from the target, which is an experimentally viable location for the pursuit of several studies related to high energy density physics. The shape of the sheath potential is crucial to the achieved high quality achromatic focusing and was achieved by exploiting the emission characteristics of protons from thicker targets, such as smaller beam divergence and ballistic emergence, as compared to tens of micron thick targets. The focusing scheme provides control on the focal length as well as chromaticity of the focusing beam by tuning the target curvature, and hence allows substantial flexibility for further beam transport.

The authors would like to acknowledge the support of and useful discussions with F. Nürnberg and M. Roth of GSI, Darmstadt, during the experiment. Authors also acknowledge funding support from EPSRC [Grants No. EP/ E035728/1 (LIBRA consortium) and No. EP/E048668/1), and the Royal Society UK, and support from K. Lancaster and the target fabrication group of RAL-STFC.

*m.zepf@qub.ac.uk

[1] M. Borghesi et al., Fusion Sci. Technol. 49, 412 (2006).

[2] M. Roth et al., Phys. Rev. Lett. 86, 436 (2001).

[3] J. Fuchs et al., Nature Phys., 2, 48 (2005).

[4] L. Robson et al., Nature Phys., 3, 58 (2006).

[5] M. Schollmeier et al., Phys. Rev. Lett. 101, 055004 (2008).

[6] S. Ter-Avetisyan et al., Laser Part. Beams 26, 637 (2008).

[7] M. Nishiuchi et al., Appl. Phys. Lett. 94, 061107 (2009).

[8] T. Toncian et al., Science 312, 410 (2006).

[9] S. Kar et al., Phys. Rev. Lett. 100, 105004 (2008).

[10] S. C. Wilks et al., Phys. Plasmas 8, 542 (2001).

[11] M. Borghesi et al., Phys. Rev. Lett. 92, 055003 (2004).

[12] P. K. Patel et al., Phys. Rev. Lett. 91, 125004 (2003).

[13] R. Snavely et al., Phys. Plasmas 14, 092703 (2007).

[14] S. Kar et al., High Energy Density Phys. 4, 26 (2008).

[15] L. Romagnani et al., Phys. Rev. Lett. 95, 195001 (2005).

[16] J. Fuchs et al., Phys. Rev. Lett. 91, 255002 (2003).

[17] D. C. Carroll et al., Phys. Rev. E 76, 065401 (2007).

[18] S. Kahaly et al., Phys. Rev. Lett. 101, 145001 (2008).

[19] L. Cao et al., Phys. Plasmas 17, 043103 (2010).

[20] J. Green et al., New J. Phys. 12, 085012 (2010).

[21] P. McKenna et al., Laser Part. Beams 26, 591 (2008).

[22] X. H. Yuan et al., New J. Phys. 12, 063018 (2010). 